\title{
Gamma detector response simulation inside the pedestal of Fukushima Daiichi Nuclear Power Station
}

\author{
Eka Sapta RIYANA*, Keisuke OKUMURA**, Kenichi TERASHIMA**, Taichi MATSUMURA** \\ and Masahiro SAKAMOTO** \\ * Collaborative Laboratories for Advanced Decommissioning Science, Japan Atomic Energy Agency \\ 2-4 Shirakata, Tokai-mura, Naka-gun, Ibaraki 319-1106, Japan \\ E-mail: riyana.eka@jaea.go.jp \\ ** Collaborative Laboratories for Advanced Decommissioning Science, Japan Atomic Energy Agency \\ Tomioka-cho, Futaba-gun, Fukushima 979-1151, Japan
}

Received: 30 October 2019; Revised: 20 February 2020; Accepted: 31 March 2020

\begin{abstract}
Prediction of the fuel debris location and distribution inside the primary containment vessel (PCV) of the Fukushima Daiichi Nuclear Power Station is important to decide further decommissioning step and strategy. The radiation measurements in the past internal investigations have not yet provided enough information to predict fuel debris location and its distribution inside PCV. To support further measurement efforts, we simulate the detector response inside the PCV and finding possible gamma radiation unique to fuel debris. The calculation result could provide useful information for future gamma detector developments.
\end{abstract}

Keywords: Fuel debris, Fukushima Daiichi Nuclear Power Station, Detector response calculation, Transport calculation, Monte Carlo simulation, PHITS code

\section{Introduction}

Prediction of the fuel debris location and distribution inside the primary containment vessel (PCV) of the Fukushima Daiichi Nuclear Power Station (1F NPS) is important to decide further decommissioning step and strategy.

It is difficult to predict the location and distribution of the fuel debris inside the PCV by the previous gamma dose rate measurements (IRID, TEPCO, 2017), because the dose rate measurements don't provide the information on wherever measured photon are originating from fuel debris or contaminated objects.

Fuel debris emit photon and neutron radiation from fission products (FPs) decay and spontaneous fission respectively. The radiations emitted from fuel debris are influenced by several factors such as fuel composition, fuel burnup before the accident, release rate of volatile FPs from the fuel at the time of the accident, etc.

In the current work, we simulate the gamma detector response inside the PCV of unit-1 by using the PHITS code (Sato et al., 2018) and finding possible gamma radiation unique to fuel debris. The calculation result could provide useful information for future gamma detector developments or for further 1F NPS fuel debris study.

\section{Calculation method}

\subsection{Calculation flow}

Figure 1 shows the calculation flow to obtain the detector response. The calculation began with estimating nuclear fuel compositions, they are, the quantity of FPs and heavy nuclides before the accident. The next step continues with the identification of the photon sources originated directly from fuel debris and the other photon sources caused by the neutron capture reactions. Finally, we calculated, in various conditions, the detector responses which are defined as the energy deposition in a detector material using the PHITS code. 


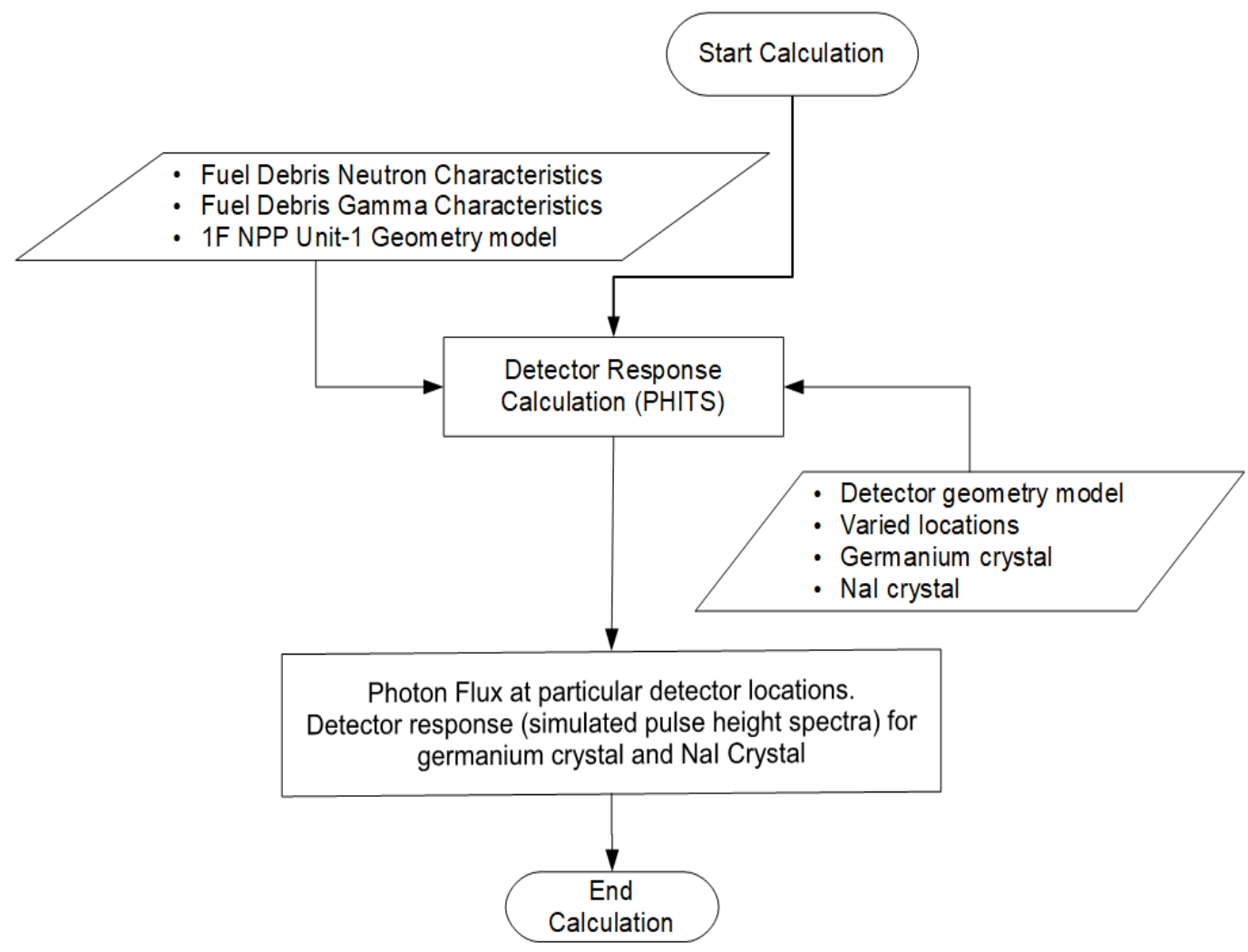

Fig.1 Calculation flow to obtain the detector response in PCV of $1 \mathrm{~F}$.

\subsection{Fuel debris compositions and radiation sources}

Information on the characteristics of gamma and neutron emitted from fuel debris were provided by previous work (Okumura K., et al., 2019; Riyana E.S., et al., 2019). The fuel debris composition was generated with several processes: First, a three-dimensional fuel inventory calculation was carried out with consideration to axial void and burnup distributions by using the reactor analysis code system MOSRA (Okumura K., et al., 2012) and its nuclear data library based on JENDL-4.0 (Shibata K., et al., 2011). Second, the activation calculation for the structural materials of a fuel assembly were carried out in consideration of impurities using ORLIBJ40 (ORIGEN2 and its revised library with JENDL-4.0) (Okumura K., et al., 2013).

The fuel debris composition described above consisted mainly of FPs, activated nuclides, structure materials and heavy nuclides such as uranium and plutonium isotopes. Noble gases and other volatile elements such as $\mathrm{Xe}, \mathrm{Kr}$ and $\mathrm{Cs}$ were removed from the fuel debris to simulate the release of them at the time of the accident. Quantity of removed FPs are shown in Table 1. The release rate were based on the result of the FP release experiment Phebus FPT4 (Bottomley P.D.W., et al., 2006).

Gamma sources in the current calculation are a result of the summation of all photon radiation produced from FPs decay. The major FPs nuclides gamma sources shown in Fig.2. One can see even after significant release, the gamma ray intensity of Cs-137 (Ba137m) is still the highest emitted photon from fuel debris. In PHITS code input we modeled all FPs gamma sources are distributed equally in the fuel debris.

Neutrons are emitted due to the spontaneous fission of heavy nuclide (e.g., $\mathrm{Pu}, \mathrm{U}$, and $\mathrm{Cm}$ ). However, total number of neutron sources are influenced by subsequent neutron-induced fission of fissile material in fuel debris in addition to the spontaneous fissions. As a result of the neutron transport, photons are emerged due to (n, $\gamma)$ or $(\mathrm{n}, \mathrm{f})$ reactions. These can be simulated by the neutron-photon coupling transport calculation by PHITS. Therefore, we calculate the fuel debris neutron spectra to predict the contribution from $(\mathrm{n}, \gamma)$ reaction. 


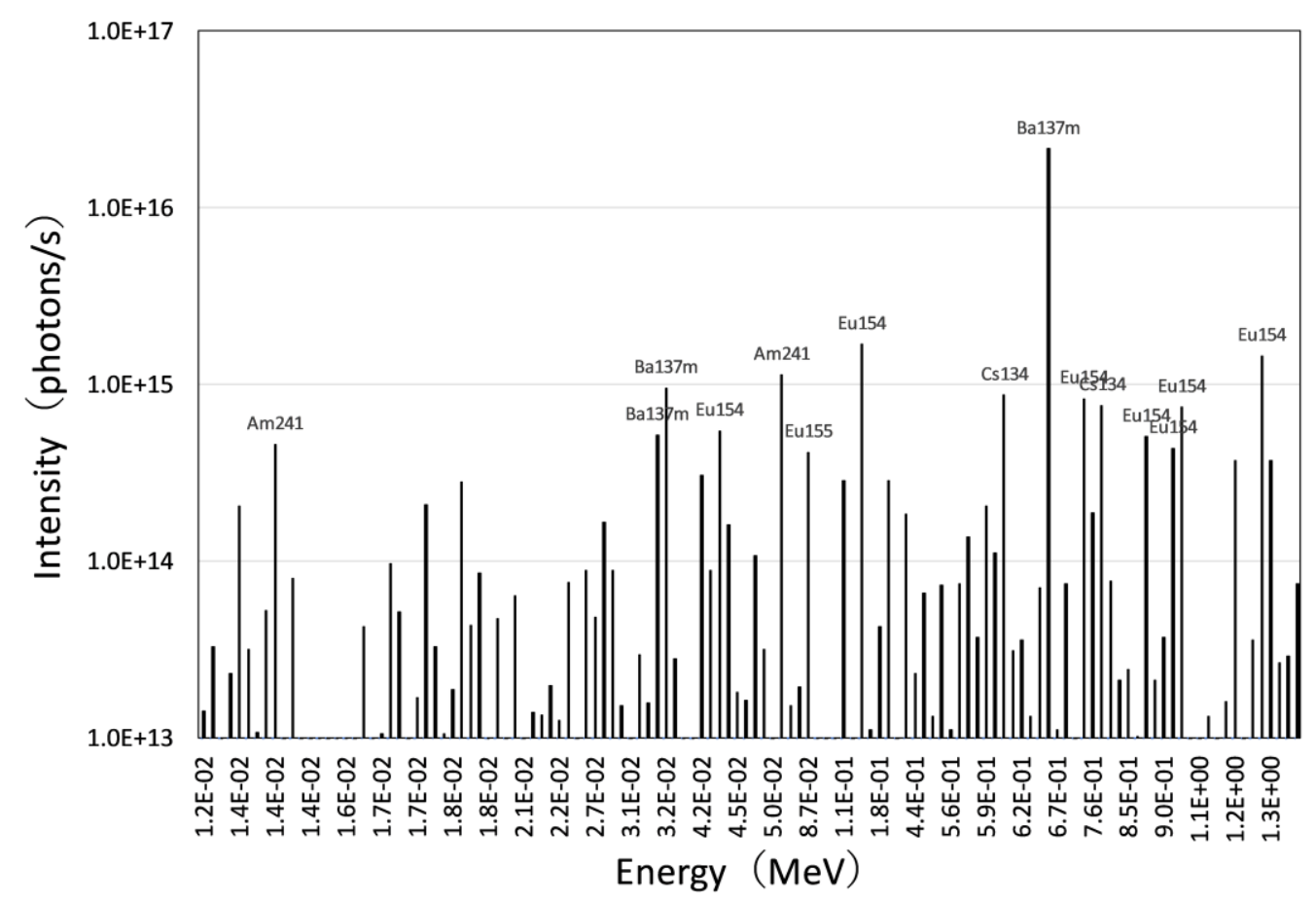

Fig.2 Unit-1 Fuel debris gamma sources.

Table 1 FPs release models

\begin{tabular}{|c|c|c|}
\hline Classification & Element & Release (\%) \\
\hline Noble Gas & $\begin{array}{c}\mathrm{He}, \mathrm{Ne}, \mathrm{Ar}, \\
\mathrm{Kr}, \mathrm{Xe}, \mathrm{Rn}\end{array}$ & 99 \\
\hline \multirow{13}{*}{ Volatile FPs } & I & 97 \\
\hline & Cs & 84 \\
\hline & $\mathrm{Te}$ & 80 \\
\hline & Mo & 77 \\
\hline & $\mathrm{Rb}$ & 53 \\
\hline & $\mathrm{Cd}$ & 44 \\
\hline & $\mathrm{Ba}$ & 35 \\
\hline & $\mathrm{Sb}$ & 30 \\
\hline & $\mathrm{Pd}$ & 27 \\
\hline & $\mathrm{Ag}$ & 9.2 \\
\hline & $\mathrm{Tc}$ & 7.1 \\
\hline & $\mathrm{Ru}$ & 1.8 \\
\hline & $\mathrm{Sr}$ & 1.4 \\
\hline
\end{tabular}




\subsection{Geometry model used in calculation}

Figure 3 shows a calculation model of the fuel debris and its vicinity inside the PCV of Unit-1. In the figure, fuel debris exists inside and outside the pedestal and they are submerged in the coolant water. Although the location of actual fuel debris are not clear yet, this situation was assumed on the basis of the information from the internal investigation and the accident progression analysis performed by the International Research Institute for Nuclear Decommissioning (IRID) (IRID, TEPCO, 2017). Although the erosion of concrete by fuel debris at the bottom of PCV is ignored for simplicity, this is not essential in this study because it is expected the radiation in the deep positions are self-shielded by heavy fuel debris covering the surface.

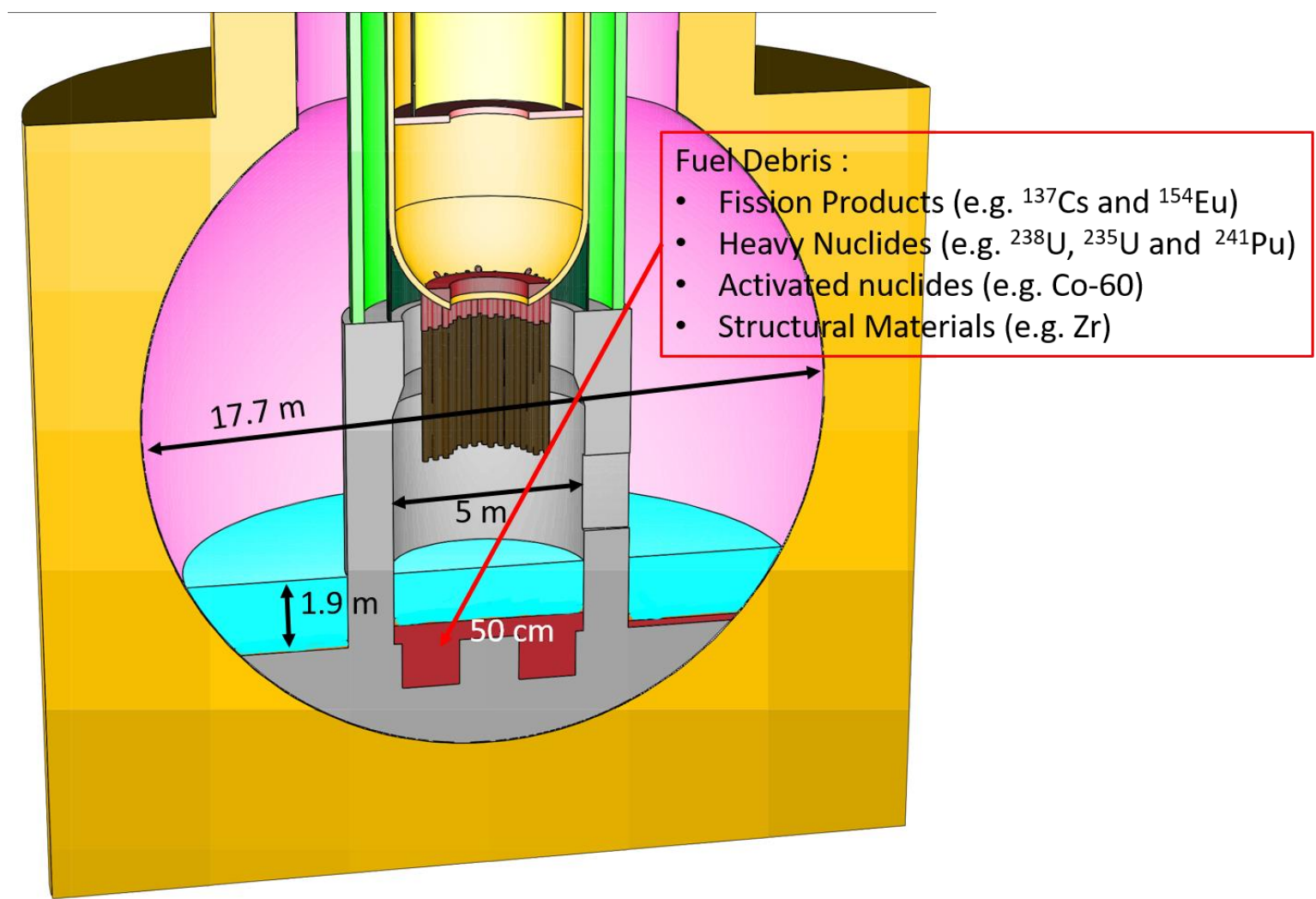

Fig.3 Geometry model of the lower part of PCV in Fukushima NPP (Unit-1).

In the current study, we selected a High Purity Germanium crystal (HPGe) as a representative of high energy resolution gamma ray detectors. It has an ability to resolved spent fuel complex photon energy spectra (Vaccaro S., et al., 2016). On the other hand, a Sodium Iodide crystal (NaI) was selected as a representative of relatively lower energy resolution detectors.

The realistic problems in utilizing these detectors in measurements inside the PCV is related to their dimensions and radiation resistances. For example, the HPGe spectroscopy system usually uses liquid nitrogen as a coolant to operate it, but it is practically difficult to bring the detector with the coolant system inside the PCV. In addition, it is not available without gamma ray shielding under the high gamma ray environments by more than $1.0 \mathrm{~Sv} / \mathrm{h}$ predicted for $1 \mathrm{~F}$ (Okumura K., et al., 2019). However, HPGe have excellent capability in gamma ray energy determination we expected the HPGe simulation would give us the ideal information to identify fuel debris inside PCV, and the NaI simulation would give us the information on necessary energy resolution.

Figure 4 shows the detector geometry model and materials used in the PHITS calculation. The detector model has 10 $\mathrm{cm}$ in diameter and $20 \mathrm{~cm}$ of length, which is based on a typical commercial n-type HPGe detector. The same geometric model was adopted for the $\mathrm{NaI}$ detector for convenience. 
The actual HPGe relative efficiency was $90 \%$ at $1.33 \mathrm{MeV}$ with reference to 3 in $\mathrm{x} 3$ in NaI(Tl) detector (Knoll G.F., 2000). The NaI crystal volume in calculation almost similar to that of the reference 3 in $x 3$ in NaI(Tl), hence its relative efficiency value is $\sim 100 \%$ at $1.33 \mathrm{MeV}$.
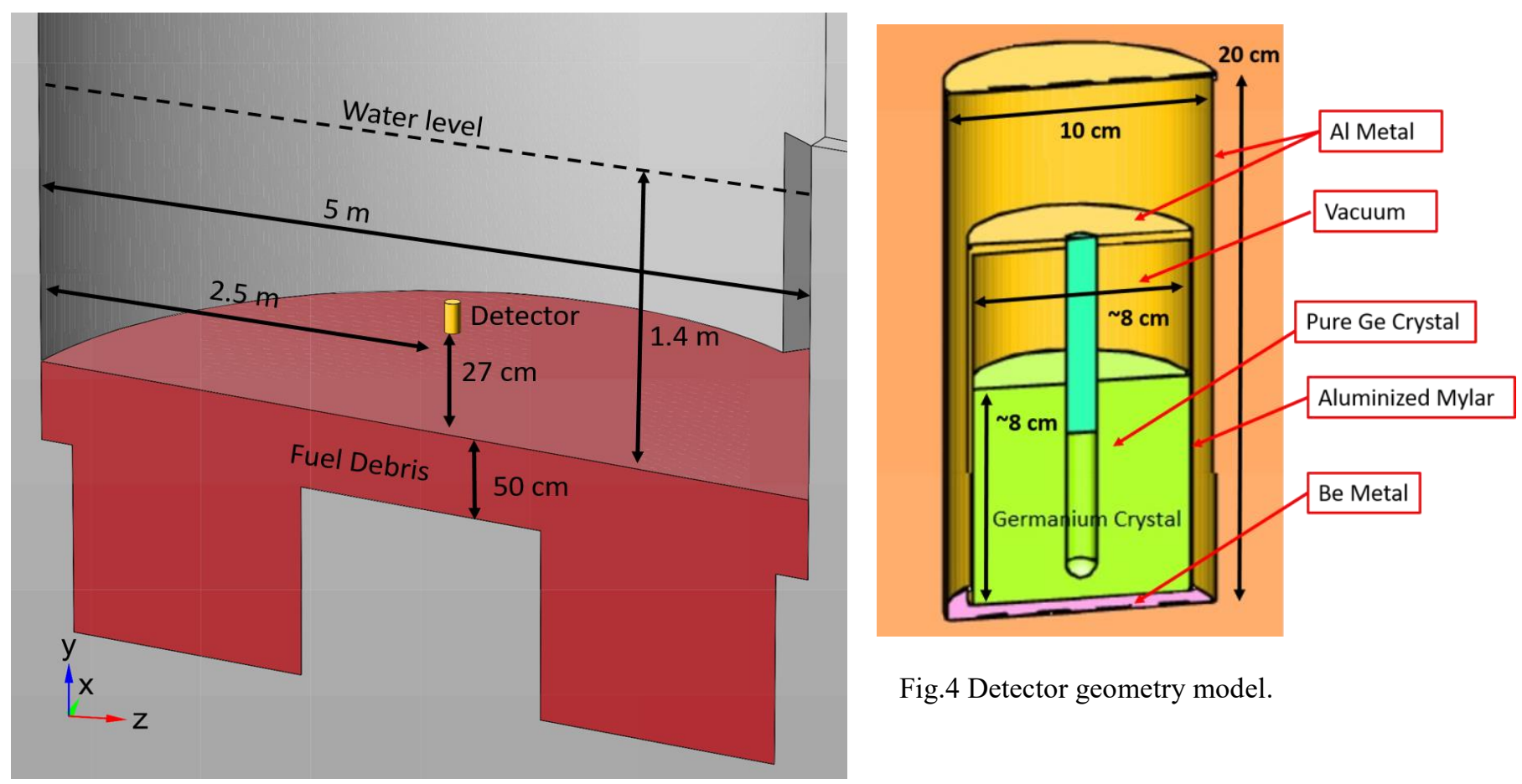

Fig.4 Detector geometry model.

Fig.5 An example of the detector position inside the pedestal.

The detector response in March 2021 (10 years after the accident) was calculated at the varied positions with different heights and radii inside the pedestal. Figure 4 illustrates an example of detector position, where water zone is not specified $\mathrm{d}$ but the detector is submerged in water. As shown in Fig.5, we assumed the fuel debris is evenly distributed inside the pedestal because of no information from the internal investigation for inside the pedestal. Since the diameter of the pedestal of Unit-1 is around $5 \mathrm{~m}$ and that of the detector is only $10 \mathrm{~cm}$, a large amount of particles histories by about $1 \times 10^{10}$ were traced in the calculations.

\section{Result and discussion}

Figure 6 shows the photon spectra calculated at the two locations with different height ( $50 \mathrm{~cm}$ apart) at the center of pedestal. The first detector location (Height 1) is at $27 \mathrm{~cm}$ above the fuel debris and the second one (Height 2) is $77 \mathrm{~cm}$ above the fuel debris. In these results, the contribution of the gamma rays from surroundings contaminated with Cs are not included, in order to make clear the contribution from the fuel debris. 


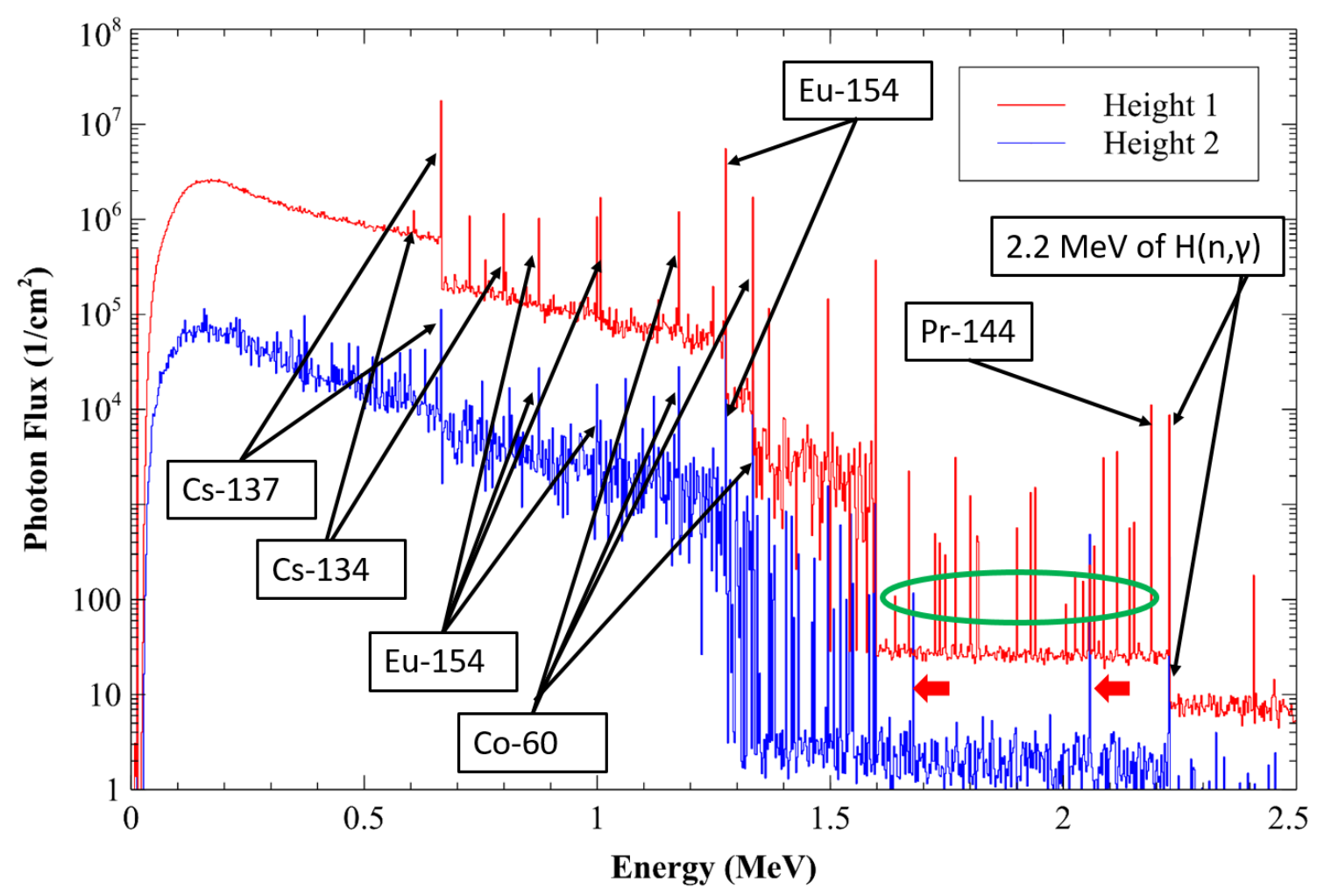

Fig.6 Photon spectra at the detector location, calculated at the center of the pedestal with two different heights: Height 1 at $27 \mathrm{~cm}$ and Height 2 at $77 \mathrm{~cm}$ above the fuel debris.

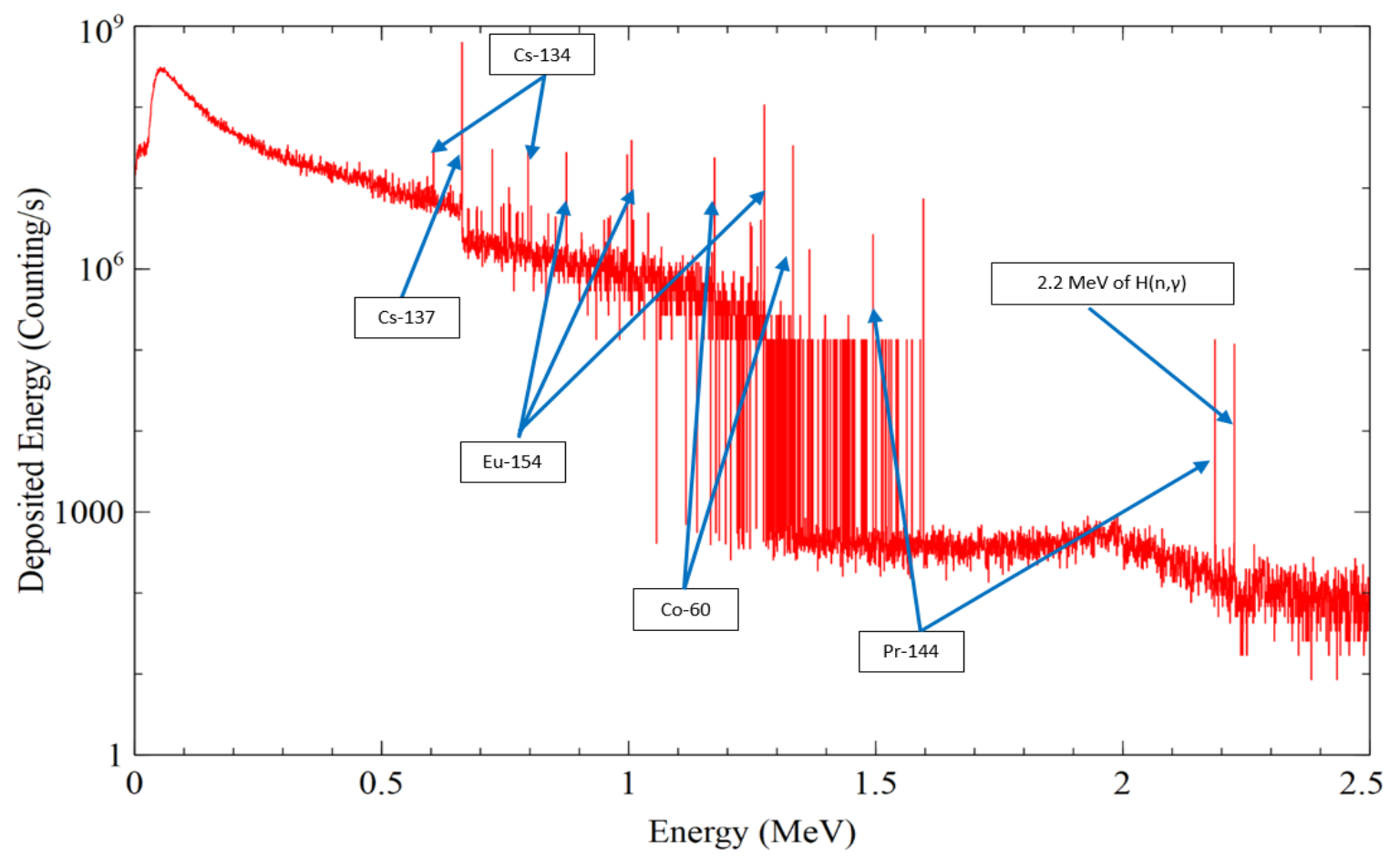

Fig. 7 Germanium detector response evaluated at the center of the pedestal and the height of $27 \mathrm{~cm}$ from the surface of the fuel debris.

Many gamma ray peaks are observed in the figure, several major gamma rays energy are indicated in Fig.6. Most of the gamma rays in Fig.6 are deposited into full-energy peaks in the Ge crystal as seen in Fig.7 (most of them are gamma 
rays from radioactive FPs). The smooth parts observed in both Fig.6 and Fig.7 are due to the Compton scatterings, however one need to differentiate between the Compton continuums observed in Fig.6 and the one in Fig.7.

The continuum photon flux observed in Fig. 6 are by-products of Compton scatterings event between gamma rays from FPs (which is discrete spectra) with fuel debris, water and other surrounding materials. Therefore, from Fig.6 we know the detector model is exposed by photon continuum spectra and gamma rays discrete spectra at the same time.

If the average size detector exposed with monoenergetic gamma ray, the typical response graph will consist of mainly a continuum and full-energy peak. The continuum produced from partial energy absorption by Compton interaction between the detector material and gamma ray and its energy ranged from almost $0 \mathrm{MeV}$ to Compton edge energy. The full-energy peak produced from full energy absorption by detector materials and has energy equal to incident gamma ray.

Therefore, the continuum observed in Fig. 7 are by-products of Compton scatterings event between photon from the outside detector (defined by Fig.6 spectra photon) and detector materials (germanium or NaI crystals). Photon sources defined by Fig. 6 has dominant in high intensity photon continuum especially below $0.8 \mathrm{MeV}$. It is obvious we also has very dominant Compton scattering event recorded in the detector response especially in lower energy part below 0.8 $\mathrm{MeV}$. PHITS code calculate deposited energy inside the detector materials, where count per second equivalent with energy deposited inside the crystal.

Generally, full-energy peaks of heavy nuclides gamma rays are appeared in the lower energy region below about $0.06 \mathrm{MeV}$, however, they are not observed in these figures because they are covered with the accumulation of Compton scatterings. If the contribution of the contaminated Cs are considered, a full-energy peak of ${ }^{137} \mathrm{Cs}$ at $0.662 \mathrm{MeV}$ and the Compton scatterings under $0.662 \mathrm{MeV}$ will drastically increase. In addition, the gamma peaks of ${ }^{134} \mathrm{Cs}$ at 0.605 and 0.796 $\mathrm{MeV}$ are still observed despite its half-life time is about 2.06 years. These are the reason that it is difficult to search for the fuel debris by measurements of gamma dose rate integrated over the whole energy.

In Fig.6, the gamma peaks are more distinct when the detector is close to the fuel debris (Height 1). On the other hand, when the detector is far away from the fuel debris (Height 2), weak gamma peaks (indicated by green ellipse) can't be detected because of gamma shielding by water. It should be noted that false gamma ray peaks (indicated by red arrows) due to the lack of statistical accuracy are observed in the case of 'Height 2'. Therefore, with consideration of large Compton continuum to detect the fuel debris in PCV, it is necessary to detect the gamma peaks higher than about 0.8 $\mathrm{MeV}$. From this point of view, the gamma peaks of ${ }^{154} \mathrm{Eu}$ and ${ }^{144} \mathrm{Pr}$ are candidates to find the fuel debris because these lanthanoids can be expected to coexist with the uranium in the fuel debris. ${ }^{154} \mathrm{Eu}$ has a half-life of 8.6 years, while ${ }^{144} \mathrm{Pr}$ is a radiation equilibrium daughter nuclide of ${ }^{144} \mathrm{Ce}$ with a half-life of 285 days. Therefore detection of ${ }^{154} \mathrm{Eu}$ is a better marker to search for fuel debris for a long time.

We can see a gamma ray peak at $2.22 \mathrm{MeV}$. This is a neutron capture gamma due to the ${ }^{1} \mathrm{H}(\mathrm{n}, \gamma)$ reaction in water. To search for the submerged fuel debris, this may be a candidate of the fuel debris marker.

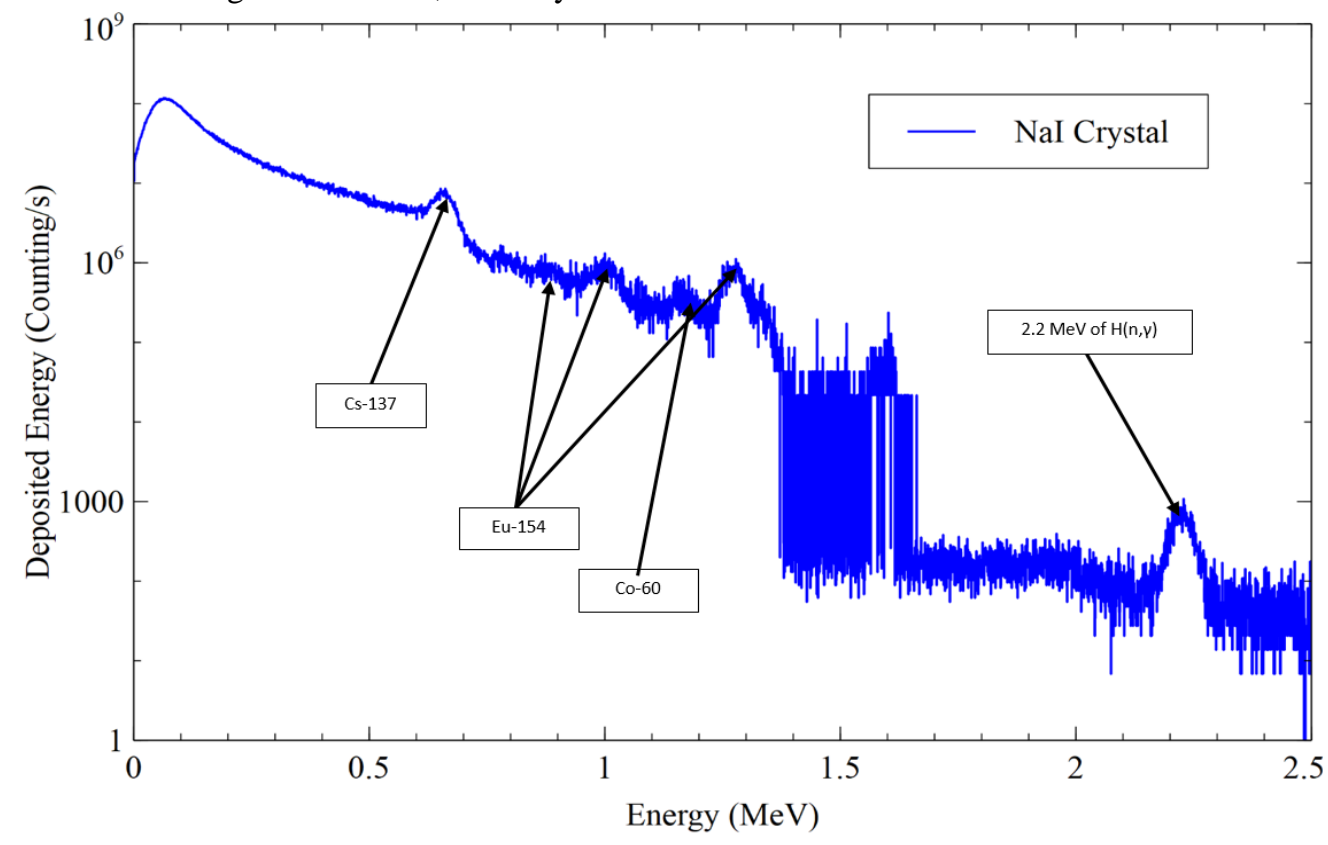

Fig. $8 \mathrm{NaI}$ detector response evaluated at the center of the pedestal with $27 \mathrm{~cm}$ height. 
The NaI detector response is shown in Fig.8. The gamma peaks of ${ }^{154} \mathrm{Eu}$ and the neutron capture gamma are observed. We can say for well separated fuel debris marker there is a possibility to use a detector with relatively lower energy resolution like a $\mathrm{NaI}$ detector.

\section{Conclusions}

In the PCV of $1 \mathrm{~F}$ where $\mathrm{Cs}$ is existed everywhere high dose rate reading not necessarily mean in the immediate presence of fuel debris, hence it is difficult to search for the fuel debris by the conventional dose rate measurements. To overcome this problem, we have to detect the radiations unique to the fuel debris. They are the gamma rays from capture of spontaneous fission neutrons emitted from actinides like ${ }^{244} \mathrm{Cm}$, or gamma rays distinguishable from those of radioactive Cs. In this study, a gamma detector simulations in PCV was carried out to obtain information useful for future gamma detector developments. As a result, it was found that the gamma peaks of ${ }^{154} \mathrm{Eu}$ and ${ }^{144} \mathrm{Pr}$ at the energy region more than $0.8 \mathrm{MeV}$ were candidates to search for the fuel debris. In the case that fuel debris are submerged, the neuron capture gamma at $2.22 \mathrm{MeV}$ is also a candidate.

\section{References}

Bottomley P.D.W., et al., Fission product and Actinide Release from the Debris Bed Test Phebus FPT4: Synthesis of the Post Test Analyses and of the Revaporisation Testing of the Plenum Samples, Nuclear Engineering and Technology. Vol.38, No.2 (2006), pp.163-174.

IRID, TEPCO, 2017, Unit 1 Primary Containment Vessel Internal Investigation. Japan: IRID TEPCO; 2017 March.

Knoll Glenn F., Radiation detection and measurement, $3^{\text {rd }}$ edition (2000), John Wiley and Sons.

Okumura K., et al., Nuclear Data for Severe Accident Analysis and Decommissioning of Nuclear Power Plant. Proc. 2012 Symposium on Nuclear Data; (2012) Nov. 15-16; Kumatori (Japan). JAEA-Conf-2013-002, pp.15-20.

Okumura K., et al., A Set of ORIGEN2 Cross Section Libraries Based on JENDL-4.0: ORLIBJ40. Japan: Japan Atomic Energy Agency (2013), Report no. JAEA-Data/Code 2012-032 (in Japanese).

Okumura K., et al., A method for the prediction of the dose rate distribution in a primary containment vessel of the Fukushima Daiichi Nuclear Power Station. Progress in Nuclear Science and Technology, Vol.6, (2019), pp.108-12.

Riyana E.S., Okumura, K. and Terashima, K., Calculation of gamma and neutron emission characteristics emitted from fuel debris of Fukushima Daiichi Nuclear Power Station, Journal Nuclear Science and Technology, Vol.56, Issue 910 (2019), pp.922-931.

Sato, T., Iwamoto, Y., Hashimoto, S., et al., Features of Particle and Heavy Ion Transport code System (PHITS) version 3.02, Journal Nuclear Science and Technology, Vol.55, Issue 6 (2018), pp.684-690.

Shibata K., et al., JENDL-4.0: A new library for nuclear science and technology, Journal Nuclear Science and Technology, Vol. 48, Issue 1 (2011), pp.1-30.

Vaccaro S., et al., PWR and BWR spent fuel assembly gamma spectra measurements, Nuclear Instruments and Methods in Physics Research A., No. 833 (2016), pp. 208-225. 